\title{
Effect of different reagents on stability of Alkalinity silica sol
}

\author{
Wang Juan ${ }^{1,}$, Niu Xin Huan ${ }^{1, b}$, Sun ming ${ }^{1, c}$, Wang $\mathrm{Ru}^{1, \mathrm{~d}}$,Liu Yu Ling ${ }^{1, \mathrm{e}}$ \\ ${ }^{1}$ IInstitute of Microelectronics, Hebei University of Technology, Tianjin, 300130,China \\ aEmail:xruyue@hebut.edu.cn, bemail:nxh@hebut.edu.cn, cemail: sunming@163.com, \\ demail:wangru@hebut.edu.cn, ${ }^{\mathrm{e} e m a i l: ~ I y l @ h e b u t . e d u . c n ~}$
}

Keywords: reagents, silica sol, stability

\begin{abstract}
In recent years, the silicon sol has now entered a relatively smooth period, and plays an important role in industries. Especially the silicon sol is used in the ultra precision Machining Technique. The most application is be the composition of slurry. Its stability is very important in the process of chemical mechanical polishing. The addition of reagent can change the $\mathrm{pH}$ and the potential of silica sol. So the different reagents are selected to improve the stability of silica sol. The results presented here show that surfactant be selected has a strong consequence on the stability of silica dispersion. It can improve the stability of silica sol. But the organic alkali and the chelator cannot improve the stability of silica sol.
\end{abstract}

\section{Introduction}

Chemical mechanical polishing technology (abbreviation: CMP) was first put forward by Monsanto [1] in 1965. With the development of micro-electronics, the CMP is proved to be the only process technology. So the study about CMP is increasing. The slurry is an important factor to affect CMP effect besides equipment and process condition. The slurry classes, physics and chemistry characteristics, particle size\& dispersion degree have strong relations with grinding effect. At present the slurry of CMP includes $\mathrm{SiO}_{2} 、 \mathrm{CeO}_{2}$ and $\mathrm{Al}_{2} \mathrm{O}_{3}$ mainly and is applied in different fields respectively. The $\mathrm{SiO}_{2}$ slurry is easy to clean because of low viscosity and weak adhesion. So the $\mathrm{SiO}_{2}$ is promising greatly.

At present the slurry of CMP includes $\mathrm{SiO}_{2}$ is applied in different fields respectively. But silicon sol is not enough to meet the requirement of CMP because of its instability. In this paper the different reagent are used to study the stability of silicon sol and the experiments are as follows.

\section{Experiments and analysis}

The reagent can make the abrasive in the polishing slurry to keep suspension, and disperse the particles, which avoid the slurry coagulation. In addition, the polishing process is also asked to add the reagent to achieve the preference adsorption after polishing. That can get good polishing effect.

Therefore, in order to explore the role of reagent on the stability of silicon sol, the different reagents are added to the silicon sol. The variation of Zeta potential and $\mathrm{pH}$ value are studied.

The relation between the stability of silicon sol and the Zeta potential and $\mathrm{pH}$ value is described in the documents domestically and internationally. In general,the silica sol is stable when the $\mathrm{pH}$ value is more than 8 . The silica sol is also stable when the absolute of the Zeta potential is above 30mv. The $\mathrm{pH}$ of silica sol studied is 10.23 , and its Zeta potential is $-40.6 \mathrm{mv}$.

The different reagents are added to the silicon sol in study.

First, the organic alkali is added to the silica sol and the Zeta potential and $\mathrm{pH}$ value are measured. The organic alkali is a weak base and it can change the alkalinity of silica sol slowly. The experiment results can be seen from fig. 1 to fig. 2 . 


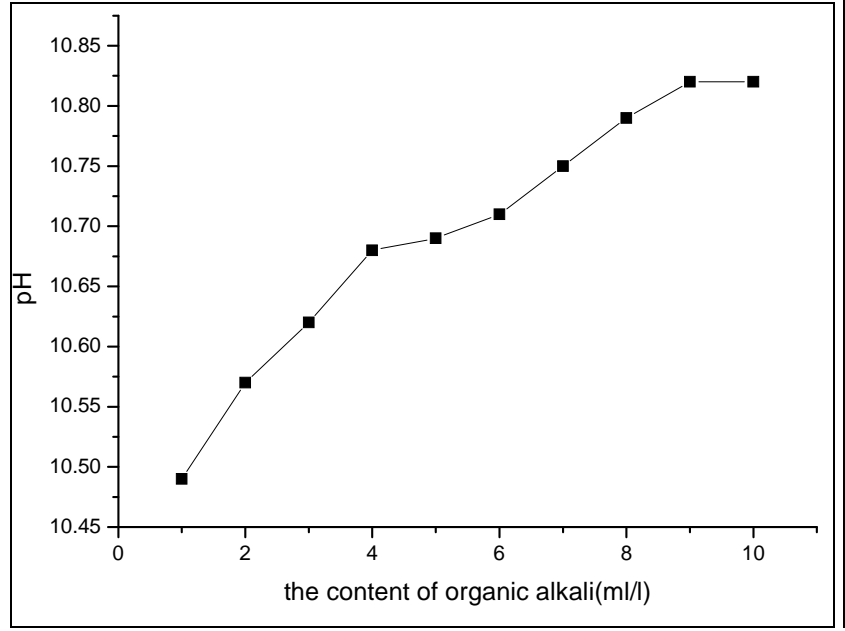

Fig.1 Effect of the organic alkali on the $\mathrm{pH}$ of silica sol

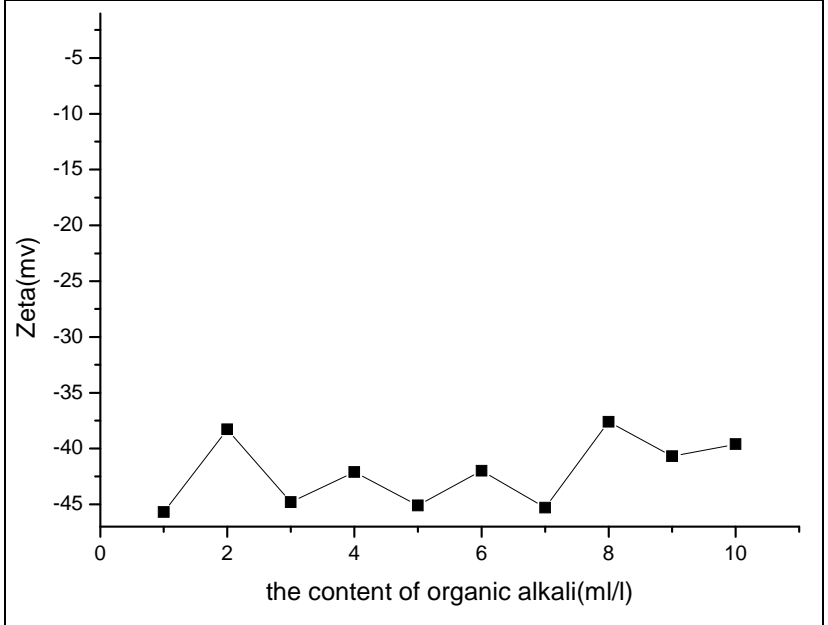

Fig.2 Effect of the organic alkali on the potential of silica sol

Fig. 1 shows the $\mathrm{pH}$ is changed greatly from 10.23 to 10.82 . With the content of the organic alkali creasing,the alkalinity is stronger. But the $\mathrm{pH}$ always is more than 8 , so the silica sol is stable viewed from this angle.

Fig.1 shows the Zeta potential does not change obviously with organic alkali amount increasing. Even if the content of organic alkali is vast, the Zeta potential does not vary. So the content of the organic alkali has little influence on the stability of silica sol.

Secondly, the two chelating agents are added to silica sol respectively. And the Zeta potential and $\mathrm{pH}$ value are measured. The chelating agent can surround the metal ion into the interior by the strong function between the chelating agent molecules and metal ions. That can prevent the metal ions play a role. In the process of CMP, the metal ions are produced usually. And these can pollute the wafer. So the chelating agent is added into the slurry. Therefore the stability of silica sol is studied by adding two chelating agents in this paper. The contrast experiment results can be seen from fig.3 to fig.6.

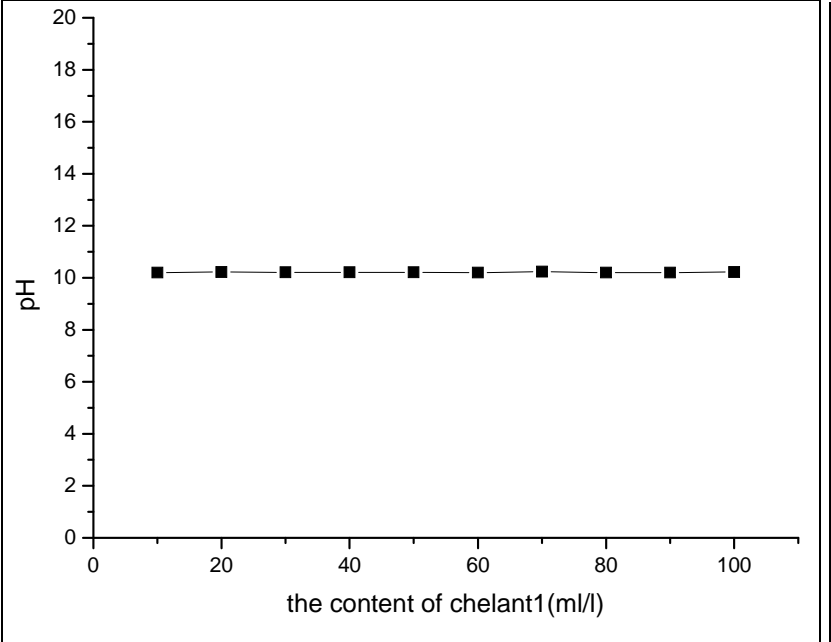

Fig.3 Effect of the first chelating agents on the $\mathrm{pH}$ of silica sol

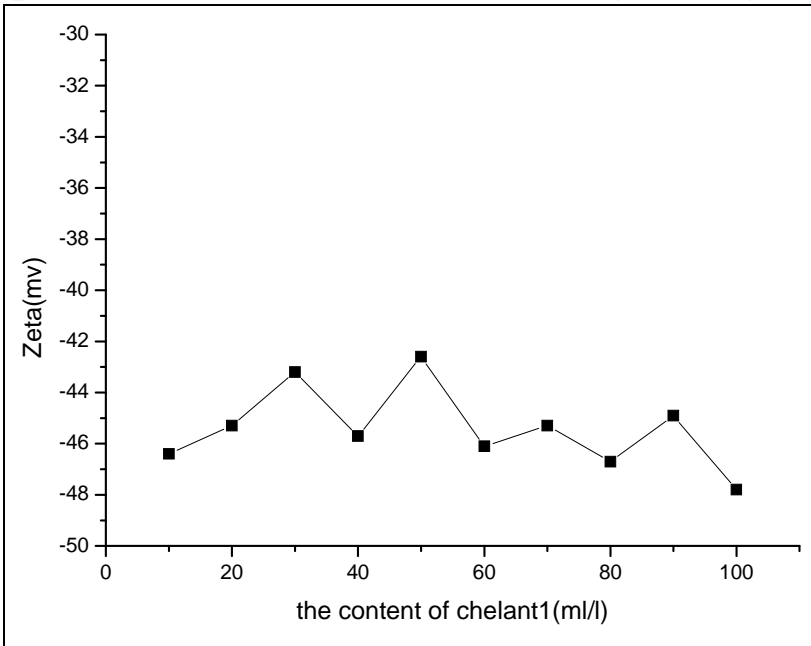

Fig.4 Effect of the first chelating agents on the potential of silica sol 


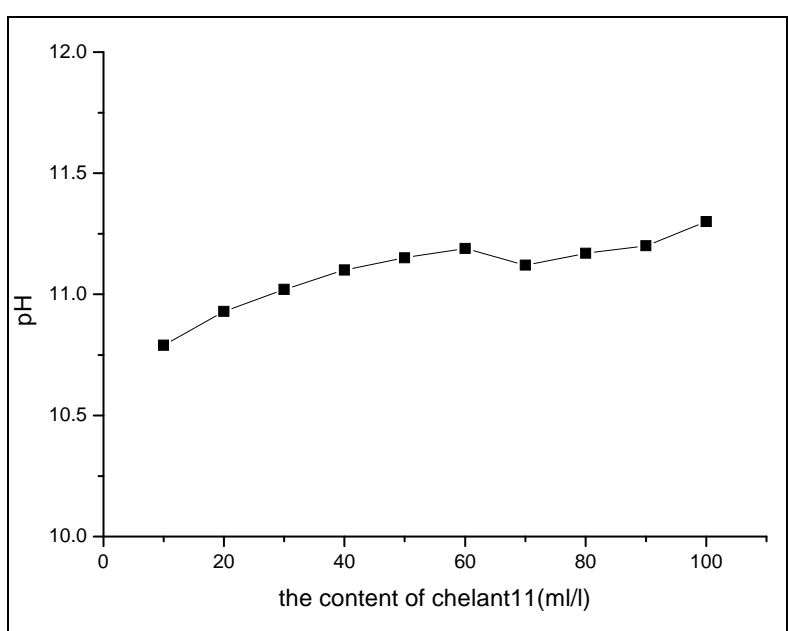

Fig.5 Effect of the second chelating agents on the $\mathrm{pH}$ of silica sol

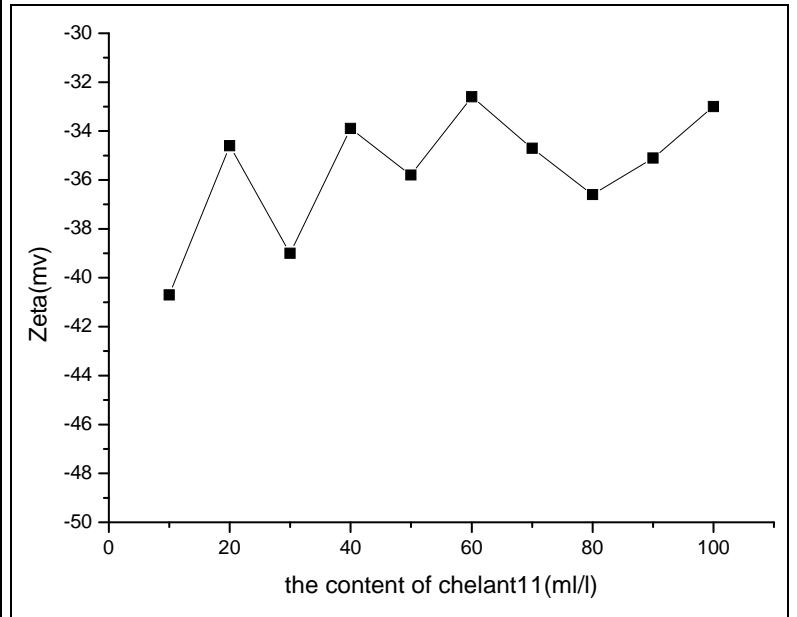

Fig.6 Effect of the second chelating agents on the potential of silica sol

It can be seen from the Fig.3 and the Fig.4, the chelating agent I has little influence in pH of silica sol. But the chelating agent has great influence in the potential of silica sol. The absolute of potential is bigger than the absolute of potential of original sample. So the chelating agent I can improve the stability of silica sol.

It can be seen from the Fig. 5 and the Fig.6, the chelating agent II has great influence in $\mathrm{pH}$ and the potential of silica sol. The $\mathrm{pH}$ arrives to 11.3. And the absolute of potential is smaller than the absolute of potential of original sample. So the chelating agent II can reduce the stability of silica sol.

Therefore, the effects of different chelating agent are different to silica sol. When the chelating agent is selected to add to slurry, we should select the chelating agent which can improve the stability of silica sol. In this way, we can gain the good effect in CMP.

In the last, the active agent is added to silica sol. The active agent can be used to modify the surface. So it is used widely to add to slurry. Then the effect of the active agent is studied in the paper. The experiment results can be seen from fig.7 to fig.8.

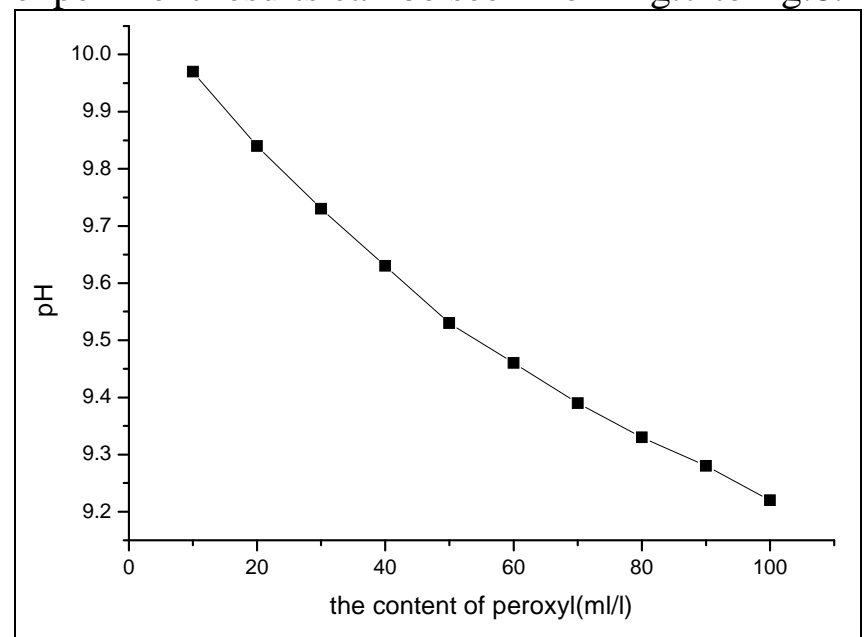

Fig.7 Effect of the active agent on the $\mathrm{pH}$ of silica sol

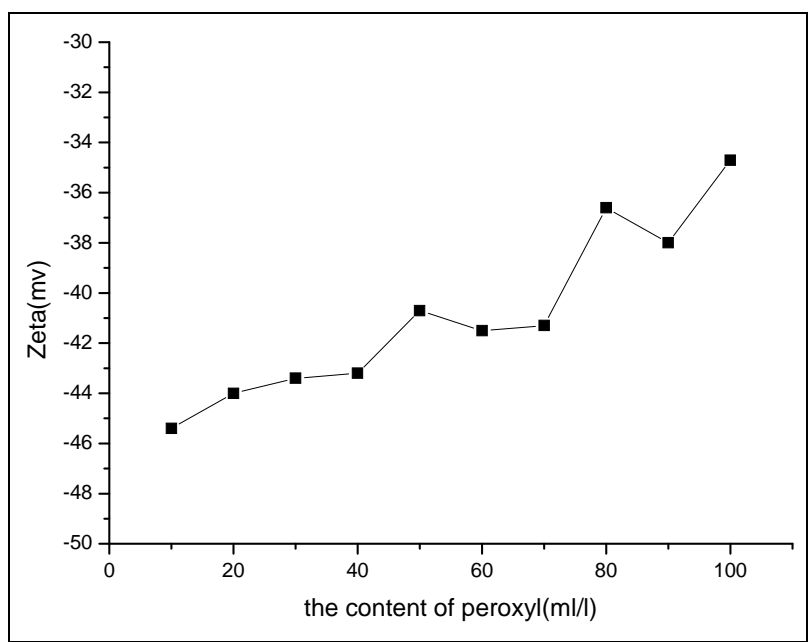

Fig.8 Effect of the active agent on the potential of silica sol

It can be seen from the Fig.7 and the Fig.8, the active agent has great influence in $\mathrm{pH}$ and the potential of silica sol. The value of is smaller than the $\mathrm{pH}$ of original sample. And the absolute of potential is bigger than the absolute of potential of original sample. So the active agent can induce the stability of silica sol. But the content of active agent is $40 \mathrm{ml} / \mathrm{l}$, the absolute of potential of silica sol is high. And the silica sol is stable.

In general, the content of the organic alkali has little influence on the stability of silica sol. And the effects of different chelating agent are different to silica sol. The suited active agent can improve the stability of silica sol. 


\section{Conclusions}

In this study, the reagent is added to silicon sols and the variation of $\mathrm{pH}$ value and the Zeta potential is studied. The suited active agent and the chelating agent selected carefully are preponderant for the stability of silicon sols. They are promising to be added to the polishing slurry.

\section{Acknowledgements}

This work was financially supported by the Major national science and technology special projects (2009ZX02308-001) and the High School of Hebei project (Z2011142).

\section{References}

[1] Walsh Robert J, Herzog Arno H. U.S. patent, U.S. Patent 3,170,273. (1965)

[2] Ming-Shyong Tsai. Journal of Materials Science and Engineering, Vol.106 (2004), p.52

[3] Anna S Z,Jan S P,Johan B. Journal of Colloids and Surfaces A:Physicochem, Vol.3 (2007), p.86

[4] Ming-Shyong Tsai,Wen-Chang Wu. Journal of Materials Letters, Vol.58 (2004), p.1881

[5] S.S.Hayrapetyan,H.G.Khachatryan. Journal of Microporous and Mesoporous Materials, Vol.78 (2005), p.151

[6] Tadafumi Adschiri,ukiya Hakuta,Kiwamu Sue,Kunio Arai. Journal of Nanoparticle Research, Vol.2-3 (2001), p.227

[7]S.M.Wang,H.Zhao,A.P.Wang,Q.N.Xun. Journal of Chemical Analysis and Meterage, Vol.16 (2007), p.4

[8]C.J.Yu,Y.L.Liao,Y.H.Lu,Z.X.Sun. Journal of University of Jinan(Sci \& Tech.), Vol.23 (2009), p.233

[9]C.Y.Qiu,K.Z.Zhang. Journal of Liaoning University of Petroleum \& Chemical Technology, Vol.25 (2005), p.1

[10] Y.Q. Fan, L.X.Chen,X.G.Bai.Liaoning Chemical Industry, Vol.39 (2010), p.281

[11]N.Q.Xu,J.X.Gu,K.Luo,S.B.Zheng.Journal of East China University of Science and Technology, Vol.29 (2003), p.642 